\title{
Electrically Induced Morphological Instabilities in Free Dendrite Growth
}

\author{
Kenneth G. Libbrecht* and Victoria M. Tanusheva \\ Norman Bridge Laboratory of Physics, California Institute of Technology 264-33, Pasadena, California 91125
}

(Received 2 March 1998)

\begin{abstract}
We describe a new instability mechanism in free dendrite growth, which arises from electrically enhanced diffusion of polar molecules near the dendrite tip. For a small applied potential, the dendrite tip velocity increases slowly with potential, as is described by an extension of normal solvability theory. Above a threshold potential, however, capillarity is insufficient to stabilize growth. We present observations that confirm this instability, which brings about a transition from enhanced normal dendrite growth to a rapidly growing needle morphology with strongly suppressed sidebranching. [S0031-9007(98)06496-5]
\end{abstract}

PACS numbers: 68.70. $+\mathrm{w}, 81.30 . \mathrm{Fb}$

The formation of stable spatial patterns is a fundamental problem in the study of nonlinear nonequilibrium systems [1]. A now-standard example of a pattern-forming system is the diffusion-limited growth of free crystalline dendrites, which are nearly ubiquitous products of rapid solidification, from either liquid or vapor precursors. Microscopic solvability theory has succeeded in furnishing a mathematically consistent and dynamically stable solution for simple 2D and 3D dendrite growth (ignoring, for example, surface kinetic and surface transport effects, both of which can be quite important in many systems) [2].

While the diffusion equation alone is sufficient to define a relation between the dendrite tip velocity and tip radius, capillarity (a typically weak but singular perturbation arising from surface tension) must be included in the theory in order to select a unique dendrite solution, and an anisotropic surface energy was found necessary for shape-preserving growth. In essence, solvability theory describes the balance between the Mullins-Sekerka instability [3], which tends to decrease the radius of curvature of a growing dendrite tip, and the Gibbs-Thomson effect of surface tension, which tends to increase it. The result in $3 \mathrm{D}$ is a dendrite roughly in the form of a paraboloid of revolution near its tip, growing at constant tip velocity, that is a solution to the equations of motion governing heat or particule diffusion in the vicinity of the solidification front $[1,2]$. Instabilities and noise amplification leading to sidebranch generation have also been well studied.

In this Letter, we introduce a new type of nonlinear dendrite instability, arising when particle diffusion is enhanced by the presence of the dendrite itself. Specifically, we consider the system of dendrite growth via vapor diffusion in a solvent gas, where the condensing particles possess a substantial electric polarizability. By applying an external electrical potential to the growing dendrite, the strong electric fields and field gradients in the neighborhood of the dendrite tip increase the particle flux onto the surface.

For small potentials, the dendrite growth is qualitatively unchanged, while electrically enhanced diffusion increases the tip velocity approximately quadratically with the value of the applied potential. This behavior is well described by the addition of an external force to the diffusion equation, resulting in an extension of normal solvability theory, which is described below.

We find, however, both theoretically and experimentally, that there exists a threshold potential for normal dendrite growth, beyond which the modified solvability theory no longer yields physical solutions. This indicates that surface tension is insufficient to stabilize the enhanced dendrite growth in this regime. Experimentally, beyond the threshold potential we observe a runaway growth of thin needle-shaped crystals, with strongly suppressed sidebranching. This behavior lies outside the realm of solvability theory, requiring mechanisms other than capillarity to determine the stable growth point.

In the presence of an external force $\vec{F}$, the diffusion equation is replaced by the Smoluchowski equation [4]:

$$
\frac{\partial c}{\partial t}=D \vec{\nabla} \cdot(\vec{\nabla} c-c \vec{F} / k T),
$$

where $c(\vec{r})$ is the solute density, and $D$ is a scalar diffusion constant. In this we have assumed that the particle mobility and diffusion constant are related via the usual Einstein relation. Ignoring interface kinetics, the continuity equation at the interface yields the normal component of the surface growth rate $v_{n}$ :

$$
v_{n}=\left.\frac{D}{c_{\text {solid }}} \hat{n} \cdot(\vec{\nabla} c-c \vec{F} / k T)\right|_{\text {surf }},
$$

where $c_{\text {solid }}$ is the solid density, and the right-hand side is evaluated at the solidification front [4].

We now consider a specific external force, namely, that which arises when an electrical potential is applied to the growing dendrite (assumed to be an electrical conductor), and the solute molecules are electrically polarizable. We assume the limit of a low solute density and unpolarizable solvent molecules, so the applied electric fields and field gradients do not depend on $c(\vec{r})$. In this case, the force $\vec{F}$ can be expressed in terms of the gradient of a 
potential, $\vec{F}=\vec{\nabla}(\vec{\mu} \cdot \vec{E})=\alpha \vec{\nabla}(\vec{E} \cdot \vec{E}) \equiv \alpha \vec{\nabla} \Phi$, where $\alpha$ is the electric polarizability. Assuming the dendrite defines an electrical equipotential surface which is nearly a paraboloid of revolution, the force potential is simply $\Phi \approx 4 \varphi_{0}^{2} \eta^{-1}(\eta+\xi)^{-1} \log ^{-2}\left(\eta_{1} / R\right)$ in parabolic coordinates, where $\varphi_{0}$ is the applied potential, $\eta=R$ defines the dendrite surface, and $\eta=\eta_{1}$ is defined by the outer limit of the experimental apparatus, which is assumed to be at $\varphi\left(\eta_{1}\right)=0$.

While techniques exist for numerically addressing the problem of dendrite growth [5], an illuminating analytic solution for the dendrite tip velocity $v$ can be obtained by assuming axial symmetry for the growing crystal and using the approximation $\Phi \approx 4 \varphi_{0}^{2} \eta^{-2} \log ^{-2}\left(\eta_{1} / R\right)$. The latter approximation formally holds only near the dendrite tip, but we believe it does not obscure any essential physics in the problem. With this, the equations separate in parabolic coordinates, giving the solution

$$
v=\frac{2 D}{R \log \left(\eta_{1} / R\right)}\left(1+\frac{R_{\mathrm{elec}}^{2}}{R^{2}}\right) \Delta_{0},
$$

where $c_{\text {sat }}=c(R)$ is the solute density near the surface, $\Delta_{0} \equiv\left[c\left(\eta_{1}\right)-c_{\text {sat }}\right] / c_{\text {solid }}$ is the supersaturation level, and

$$
R_{\text {elec }}^{2}=\left(\frac{c_{\text {sat }}}{c_{\text {solid }}}\right) \frac{4 a \varphi_{0}^{2}}{\Delta_{0} k T \log ^{2}\left(\eta_{1} / R\right)} .
$$

This solution, for which we have taken $\partial c / \partial t=0$ in the diffusion equation, is appropriate in the limit $\eta_{1} \ll \ell$, where $\ell=2 D / v$ is the diffusion length, which is the case for the experiments described below. In the limit $R_{\text {elec }} \rightarrow 0$, this reduces to the well-known Ivantsov relation for growing dendrites [6]. For moderate molecular polarizabilities, it is possible to achieve $R_{\text {elec }}>$ $R$ under reasonable experimental conditions; hence, the dendrite growth is found to be strongly influenced by the applied electrical potential.

It can be seen from this expression that the substantive effect of the electrical potential is to add an $R$-dependent term to the supersaturation, yielding an effective $\Delta_{0}^{*}(R)=\Delta_{0}\left(1+R_{\text {elec }}^{2} / R^{2}\right)$. At the same time, the applied electrical potential does not alter the surface capillarity, so physically we expect that the crystal growth will be modified primarily by the effects of this electrically enhanced supersaturation. One of the main results from 3D selection theory, however, provides that the stability parameter

$$
\sigma \equiv \frac{2 D d_{0}}{R^{2} v}
$$

is independent of supersaturation (but depends on crystalline anisotropic roughly as $\sigma \sim \epsilon^{7 / 4}$ for small $\epsilon$ ), where $d_{0}=R_{\text {crit }} \Delta_{0} / 2$ is the capillary length, proportional to the isotropic part of the surface tension, with $R_{\text {crit }}$ equal to the critical radius for homogeneous nucleation. Thus we argue on physical grounds that $\sigma$ is expected to be at most weakly dependent on $\varphi_{0}$, at a level which we will ignore in comparison to the effects of enhanced diffusion. With this assumption, we then use relation (5) from $3 \mathrm{D}$ selection theory, together with the modified Ivantsov relation (3), to select both $v$ and $R$.

Combining these yields an approximately quadratic equation for $R$ :

$$
R^{2}-R_{0} R+R_{\text {elec }}^{2}=0,
$$

where $R_{0}$ is the normal selected dendrite tip radius when $R_{\text {elec }}=0$ [since $\eta_{1} \gg R, \log \left(\eta_{1} / R\right)$ depends only very weakly on $R$ ]. For $R_{\text {elec }} \ll R_{0}$, this gives the tip radius $R \approx R_{0}\left(1-R_{\text {elec }}^{2} / R_{0}^{2}\right)$ and tip velocity $v \approx$ $v_{0}\left(1+2 R_{\text {elec }}^{2} / R_{0}^{2}\right)$. The radius decreases with increasing $R_{\text {elec }}$ until a limit is reached at $R_{\min }=R_{0} / 2$, and $v_{\text {max }}=$ $4 v_{0}$. The quadratic equation has no real roots for $R_{\text {elec }}>$ $R_{0} / 2$, indicating that the above modified solvability theory cannot be used for large $R_{\text {elec }}$.

The lack of real roots for large applied potentials is related to the familiar phenomenon of nucleation in a cloud chamber. For uncharged droplets, there exists a critical radius $R_{\text {crit }}$, below which the Gibbs-Thomson effect prevents spontaneous growth. Sufficiently charged droplets, however, can grow at all radii, which can be seen from a spherically symmetric solution to the Smoluchowski equation. In the present case of dendrite growth, above a threshold potential we find that surface tension can no longer stabilize the tip radius. At this point, the tip experiences runaway growth as $R \rightarrow 0$ under the influence of the Mullins-Sekerka instability (here enhanced by electric forces). The tip velocity thus increases until it is stabilized by some other mechanism.

An experimental demonstration of this growth instability was realized by growing ice dendrites from water vapor, in a solvent gas of ordinary air at 1-atm pressure $[7,8]$. Although surface kinetic effects are important in ice crystal formation, often producing strongly faceted crystal growth, we find at $-15 \mathrm{C}$ (the peak of the dendrite growth regime for ice) and supersaturations $\Delta_{1} \equiv\left(c_{\text {solid }} / c_{\text {sat }}\right) \Delta_{0} \gtrsim 0.4$ the prism faces are rough, resulting in dendrites with approximately parabolic tips. In this parabolic regime, the growth of the prism faces is described by the Hertz-Knudsen relation [9], and is no longer dominated by surface kinetics. Thus in this regime we expect the dendrite growth to be approximately described by the above modified solvability theory.

An electrical potential was applied after establishing the growth of a single normal $\left(\varphi_{0}=0\right)$ dendrite, which had a tip velocity of typically $v \approx 3 \mu \mathrm{m} / \mathrm{s}$; the finite conductivity of ice, along with the very low current flow [7] ensured that the dendrite possessed an equipotential surface. Occasionally the applied potential resulted in dendrites with a large uniform sidebranch spacing [8], but more typically the growth remained qualitatively similar to that of normal growth at low potentials, with $v$ increasing with $\varphi_{0}$ up to a threshold potential. An example 
of this behavior is shown in Fig. 1. A comparison of the data and theory can be made by combining Eqs. (5) and (6) above into $v=4 v_{0}\left[1+\left(1-\varphi_{0}^{2} / \varphi_{\max }^{2}\right)^{1 / 2}\right]^{-2}$, where $v_{0}=2 D d_{0} / \sigma R_{0}^{2}$ is the normal tip velocity. In Fig. 1, the two parameters $v_{0}$ and $\varphi_{\max }$ were adjusted via least squares to best fit the measured points. This fit gave $\varphi_{\max }=1450 \mathrm{~V}$, which was in reasonable agreement with the observed threshold value.

Above the threshold potential, the enhanced dendrite growth behavior became unstable. If the potential were slowly raised above threshold with $\Delta_{1} \lesssim 0.6$, the dendrite tip would sometimes make a smooth transition to a rapid growth behavior, shown in Fig. 2(a). This resulted in a thin, featureless needle-shaped crystal, with a diameter of $\sim 20-30 \mu \mathrm{m}$, moving at velocities typically $20-70 \mu \mathrm{m} / \mathrm{s}$ along the original $a$-axis direction (although velocities as fast as $200 \mu \mathrm{m} / \mathrm{s}$ were observed). The needle velocities were observed to show considerable variation, which remains inexplicable since it was not simply correlated with the external growth conditions.

If the potential was slowly raised at higher saturations $\left(0.6<\Delta_{1}<2\right)$, the dendrite tip often underwent the tipsplitting instability shown in Fig. 2(b). This resulted in a restructuring of the crystal at the dendrite tip, so that further growth occurred from an appended crystal whose [11̄00] axis was approximately collinear with the $a$ axis of the original crystal [8]. We are uncertain of the cause of this peculiar behavior, which may be related to electrofreezing phenomena [10].

If a potential value above the threshold was suddenly applied at any saturation, the result was usually the copious production of thin needle crystals, similar to that shown in Fig. 2(a), which typically appeared from the sharp corners of faceted crystals. We believe this "hyperelectric" needle growth is stabilized by heating at

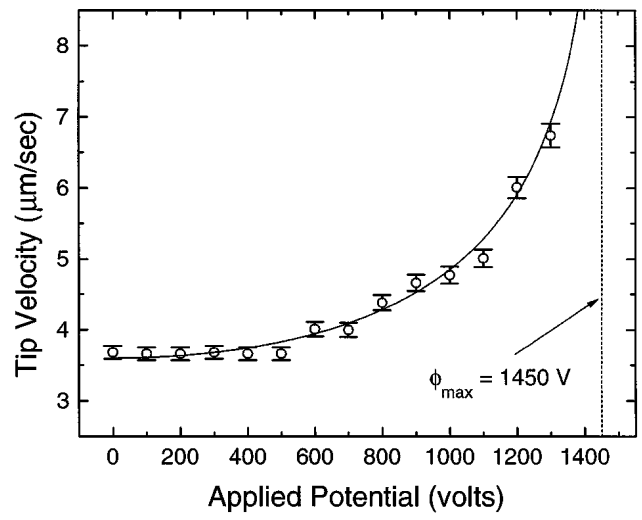

FIG. 1. Data points show measurements of the tip velocity of a single growing dendrite as a function of the applied electrical potential. The solid line is a fit to the points using the modified solvability theory described in the text. The two free parameters in the theory, $v_{0}$ and $\varphi_{\max }$, were adjusted in a leastsquares fit to the data, which gave a best fit $\varphi_{\max }=1450 \mathrm{~V}$. This dendrite underwent a tip-splitting instability when the potential was raised from 1300 to $1400 \mathrm{~V}$. the needle tip, which is substantial owing to the large latent heat of sublimation of ice. Calculations show that the degree of heating is sufficient, given dissipation via conduction to the surrounding air, to significantly raise the temperature of the growing tip and thus to halt further increase of the tip velocity.

It is conceivable that the mobility of water molecules on the growing crystal surface may be affected by the strong electric field gradients near the sharp dendrite tip [11], and thus may be responsible for some of the phenomena reported here. We believe, however, that such effects are minor in comparison to the enhanced diffusion described above. First of all, the surface fields, while substantial, are small compared to intrinsic crystal surface fields [12]. And second, we observed that none of the phenomena described here exhibited any dependence on the sign of the applied potential. This observation also allows us to effectively rule out ionization effects near the dendrite tip as playing any significant role in the growth dynamics.

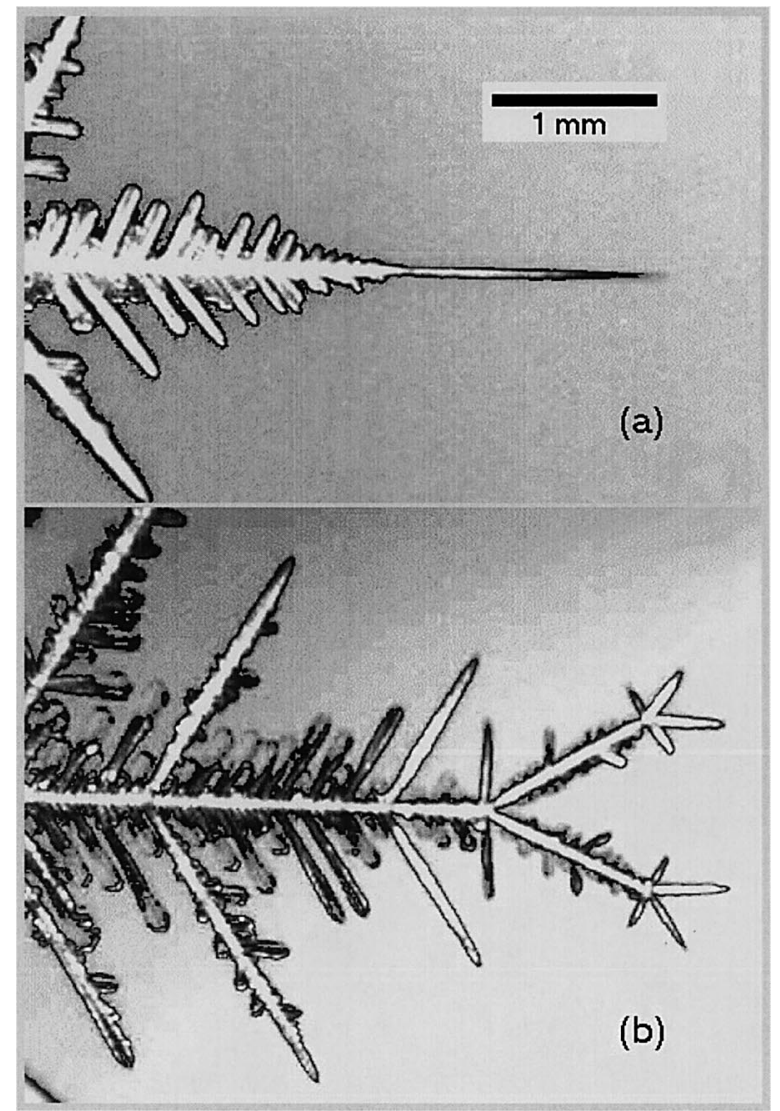

FIG. 2. Images of electrically induced dendrite instabilities, which occurred as the applied potential was slowly raised above its threshold value. Both images were passed through a solarizing filter for edge and contrast enhancement. In (a) the dendrite tip transformed from its normal form, with $v_{\text {tip }} \approx$ $3 \mu \mathrm{m} / \mathrm{s}$ and exhibiting sidebranches, to a rapid electric needle growth with $v_{\text {tip }} \approx 30 \mu \mathrm{m} / \mathrm{s}$ and suppressed sidebranching. In (b) the dendrite tip underwent a tip-splitting instability which rotated the crystal axis by $30^{\circ}$; after further growth the same instability repeated on the two advancing branches. 
In summary, we suggest a new class of dendrite growth instabilities, brought about when the presence of the dendrite itself affects the diffusion of material to its surface, adding a nontrivial external force term to the diffusion equation. Within this class, we have examined dendrite growth from polar molecules in the presence of an applied electrical potential, which is well described by a modified solvability theory. The principal result of this theory is the existence of a threshold potential, above which dendrite growth can no longer be stabilized by surface tension effects.

We note that the enhanced diffusion brought about by an applied potential is well understood at a fundamental level, and is straightforward to compute. Thus the applied potential provides the experimenter with a convenient, continuously adjustable parameter with which to alter dendrite growth. Further studies on other polar systems with less complex surface properties in comparison to ice should provide new insights into pattern formation in diffusion-limited growth. Such studies may shed light on remaining problems in understanding the dependence of the stability parameter on crystal anisotropy [13], and should also contribute to the general theory of morphological transitions during nonequilibrium growth [14]. Qualitatively similar morphological transitions are well known in studies of electrodeposition [15], but in these cases the physical mechanisms driving the transitions remain poorly understood. The transition we have described here is currently more amenable to quantitative theoretical analysis.

A particularly interesting outcome of the dendrite instability described above is the stable production of thin, featureless needle crystals, which can in principle be grown to any length. The growth dynamics of these needles is qualitatively different from needles grown via more conventional means, for example, via single screw dislocations at needle tips [16], or via the vapor-liquidsolid mechanism [17], and may be of some practical interest, particularly for large organic molecules, which can have substantial electric polarizabilities.

We acknowledge support for V.M.T. by Mr. and Mrs. Downie D. Muir III.
*Electronic address: kgl@caltech.edu

[1] For reviews, see E. Ben-Jacob, Contemp. Phys. 38, 205 (1997); 34, 247 (1993); J. S. Langer, in Chance and Matter, Proceedings of the Les Houches Summer School, Session XLVI (Elsevier, New York, 1987); H. MullerKrumbhaar, in Materials Science and Technology: a Comprehensive Treatment, edited by R. W. Cahn et al. (VCH, Weinheim, 1991); M. C. Cross and P.C. Hohenberg, Rev. Mod. Phys. 65, 851 (1993).

[2] E. Brener, Phys. Rev. Lett. 71, 3653 (1993); A. Karma and W. J. Rappel, Phys. Rev. Lett. 77, 4050 (1996); Y. Saito, Statistical Physics of Crystal Growth (World Scientific, Singapore, 1996).

[3] W. W. Mullins and R. F. Sekerka, J. Appl. Phys. 34, 323 (1963); J. S. Langer, Rev. Mod. Phys. 52, 1 (1980).

[4] S. Chandrasekhar, Rev. Mod. Phys. 15, 1 (1943).

[5] Y. Saito, G. Goldbeck-Wood, and H. Muller-Krumbhaar, Phys. Rev. Lett. 58, 1541 (1987); Phys. Rev. A 38, 2148 (1988).

[6] G. P. Ivantsov, Dokl. Akad. Nauk SSSR 58, 1113 (1947).

[7] J. T. Bartlett, A.P. van den Heuval, and B. J. Mason, Z. Angew. Math. Phys. 14, 599-610 (1963).

[8] V.M. Tanusheva and K. G. Libbrecht (to be published).

[9] E. Yokoyama, J. Cryst. Growth 128, 251 (1993); T. Kuroda and R. Lacmann, J. Cryst. Growth 56, 189 (1982).

[10] I. M. Svishchev and P. G. Kusalik, J. Am. Chem. Soc. 118, 649 (1996); M. Gavish et al., Science 256, 815 (1992).

[11] C. R. Slaughterbeck et al., J. Vac. Sci. Technol. A 14, 1213 (1996).

[12] P. V. Hobbs, Ice Physics (Clarendon, Oxford, 1974).

[13] A. Dougherty and A. Gunawardana, Phys. Rev. E 50, 1349 (1994); M. Muschol, D. Liu, and H.Z. Cummins, Phys. Rev. A 46, 1038 (1992).

[14] O. Shochet et al., Physica (Amsterdam) 187A, 87 (1992).

[15] Y. Sawada, A. Dougherty, and J. P. Gollub, Phys. Rev. Lett. 56, 1260 (1986); D. Grier et al., Phys. Rev. Lett. 56, 1264 (1986).

[16] F. C. Frank, Discuss. Faraday Soc. 5, 48 (1949); W. Miao et al., J. Mater. Sci. 32, 1969 (1997).

[17] R. S. Wagner and W.C. Ellis, Appl. Phys. Lett. 4, 89 (1964); A. M. Morales and C. M. Lieber, Science 279, 208 (1998). 